\title{
APLICAÇÃO DE SOLUÇÕES TÉCNOLOGICAS NO GERENCIAMENTO DA LOGÍSTICA INTERNA: CASO VALLOUREC *
}

Flaviana Dias Rocha Pimenta ${ }^{1}$

\section{Resumo}

Com o objetivo de gerenciar e monitorar os recursos contrados para a movimentação interna, a VSB (Vallourec Soluções Tubulares do Brasil), implementou ao longo do ano de 2016 em parceria com a empresa DDMX, algumas soluções tecnológicas de gerenciamento da frota dos equipamentos subcontratados, atendendo as necessidades de gestão do contrato, incluindo itens de segurança e produtividade. $\mathrm{O}$ presente trabalho tem como objetivo apresentar o modelo de gerenciamento e sua aplicação no dia a dia da gestão, bem como os principais benefícios com a sua implantação.

Palavras-chave: Logística Interna; Suprimentos, Sistema de Gestão, Soluções Tecnológicas.

\section{APPLICATION OF TECHNOLOGICAL SOLUTIONS IN THE MANAGEMENT OF INTERNAL LOGISTICS: CASE VALLOUREC}

\section{Abstract}

In order to manage the resources used on the internal transportation, VSB (Vallourec Soluções Tubulares do Brasil), supported by DDMX, has developed during 2016 technological solutions to improve the handle of its subcontracted fleet, including security and productivity itens. The purpose of this report is to present this management concept and its application on daily practices, as well the benefits reached with its application.

Keywords: Logistics; Supply Chain; Management System; Technologic Solutions.

1 Pós Graduada em Gestão Estratégica de Logística, Coordenadora de Logistica, Superintendência de Planejamento e Logística, Vallourec Soluções Tubulares do Brasil, Belo Horizonte, Minas Gerais, Brasil. 


\section{INTRODUÇÃO}

Atualmente as empresas tem focado sua estrutura em seu core business, com isso cada vez mais as mesmas estão contratando operadores logísticos na prestação de serviço, e para viabilizar e garantir o atendimento as demandas de forma precisa e sem excesso de recursos, a implantação de um sistema de gerenciamento de frotas é fundamental para de forma clara os gestores considerarem todas as variáveis nas tomadas de decisão e gestão de contratos.

As informações utilizadas devem ser confiáveis e automatizadas, sendo o grande desafio das organizações: definir e implementar um modelo tecnológico aderente a sua necessidade e fácil entendimento para os usuários finais. Observamos nas ultimas décadas com o uso de eletrônico e da automatização da produção, a transformação da industria 2.0 para 3.0.

Com as constantes evoluções tecnológicas aliadas as organizações, em virtude de todas as facilidades de comunição, recursos tecnológicos, manipulação de dados, onde é possível coletar e analisar dados entre maquinas, criando processos mais rápidos, flexíveis e eficientes a custos reduzidos, iniciasse a quarta revolução industrial, sendo denominado a Indústria 4.0.

Com o papel que a logística exerce na indústria, a utilização de ferramentas de TI para a evolução em paralelo com todo o processo produtivo, é fundamental para garantir o sucesso das organizações. Conforme Laudon \& Laudon (2007), a tecnologia de informação pode ser definida como um conjunto de hardware e software utilizados para uma empresa alcançar seus objetivos. Para Rezende (2008), a tecnologia da informação define-se como sendo o conjunto de métodos e ferramentas que facilitam as estratégias e tomadas de decisões, tornando possível a adequada compreensão de toda a situação de forma rápida e precisa.

Com isso, o uso da tecnologia disponível no mercado e a escolha de uma empresa que atenda as demandas e ofereça soluções aos problemas específicos da sua operação, com constantes inovações e ferramentas práticas e de fácil aderência a todas as particularidades o dia a dia, garantem a confiança nas tomadas de decisão e no atendimento as regras estabelecidas entre as empresas envolvidas na cadeia logística: industria e prestador de serviço.

\section{MÉTODOS}

A Vallourec Soluções Tubulares do Brasil (VSB) é uma das mais modernas siderúrgicas integradas do mundo, produz tubos de aço sem costura a partir de matéria-prima e energia fornecidas pelas subsidiárias Vallourec Florestal Ltda e Vallourec Mineração Ltda. Um processo de produção autossustentável que credencia os seus produtos como Tubos Verdes.

A gerência de logística interna e expedição de produtos da VSB é a responsável por toda movimentação interna de tubos acabados dentro da usina barreiro, incluindo o transporte de tubos entre unidades de produção, equipamentos de manipulação de tubos nos pátios e nas área de produção e pelos equipamentos de apoio as áreas de manutenção e produção (não produto). Os fluxos de transporte se concentram 
em uma área de aproximadamente $1.200 .000 \mathrm{~m}^{2}$, envolvendo 120 pontos de carga e descarga diferentes de produtos, conforme figura 1.

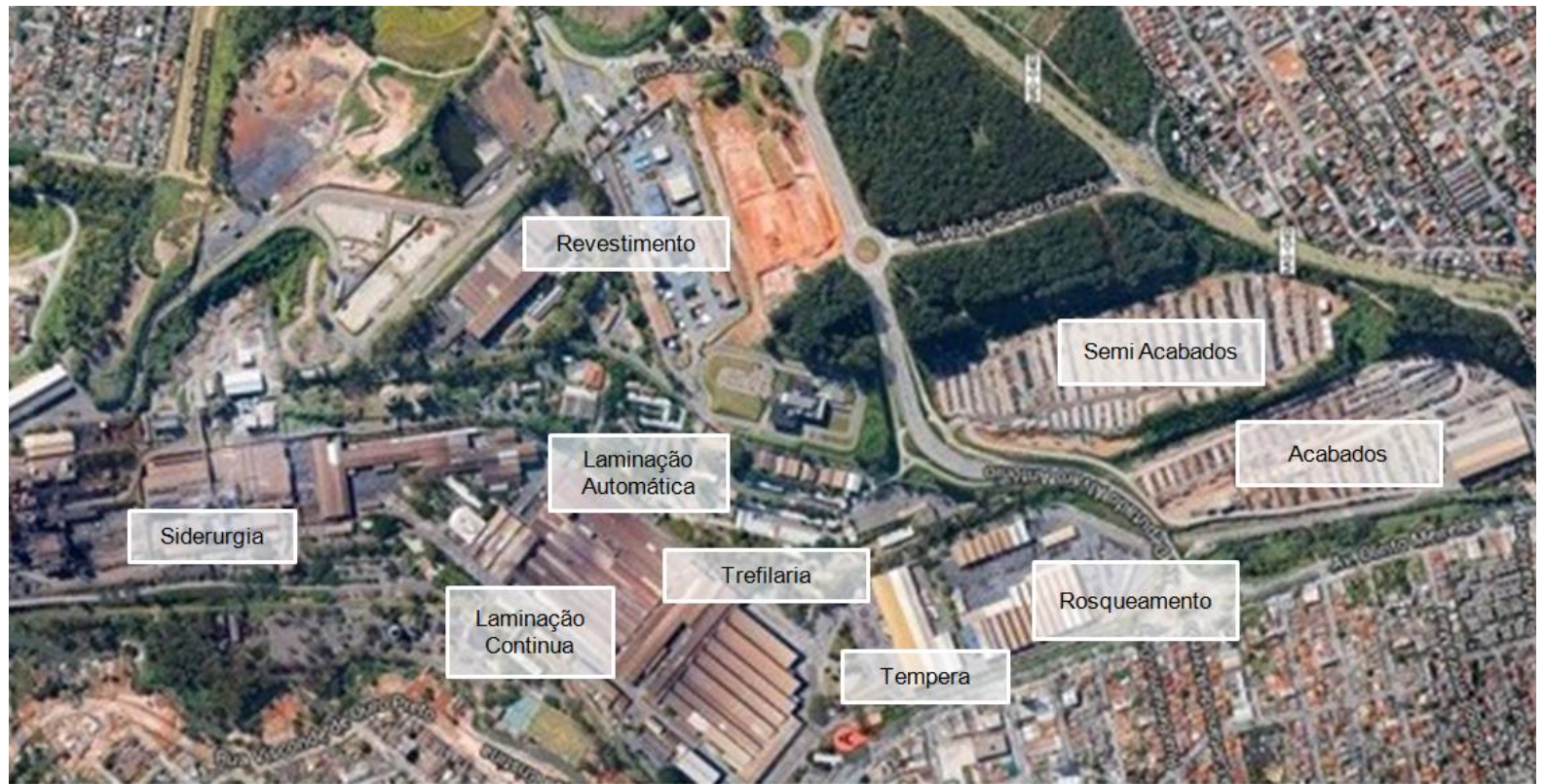

Figura 1 - Entradas e Saídas para movimentação interna

\subsection{Descrição do Problema}

Devido à complexidade da movimentação interna de materiais, entre cerca de 120 pontos de carga e descarga, a VSB possui atualmente cerca de 104 equipamentos dedicados para esta atividade.

Anteriormente havia a gestão apenas de alguns tipos de equipamentos, carretas e tratores que representava cerva de $48 \%$ dos recursos, através de uma central gerenciada pela VSB que recebia as solicitações via sistema e alocava as atividades para o operador logístico, onde a única funcionalidade do sistema era 0 mapeamento dos equipamentos na planta da Usina Barreiro.

A tecnologia anteriormente utilizada não possibilitava a gestão de outros indicadores e de aplicações de telemetria no processo de gestão, e era utilizada a tecnologia de comunicação via antena de rádio, restrita apenas a raio de ação das mesmas, e com inúmeras limitações. Em 2015, foi realizado uma renovação de contrato com uma única empresa, onde foram incorporados diversos indicadores de gestão que eram fundamentais para a otimização e produtividade dos recursos, que sem a uma forma automatizada de apuração de dados seria moroso e inviável de controlar.

Outra grande dificuldade na gestão dos recursos anteriormente era a interface (figura 2) não amigável do sistema, as limitações na implantação do mesmo sistema para toda a frota e manter as características de cada atividade individualmente, a dependência de hardwares exclusivos para a operacionalização do sistema, a necessidade de servidores locais e um único computador central para operação da gestão, e a necessidade de apontamentos manuais pelo operador dos equipamentos, o que poderia prejudicar as analises de ocupação e produtividade de cada equipamento. 


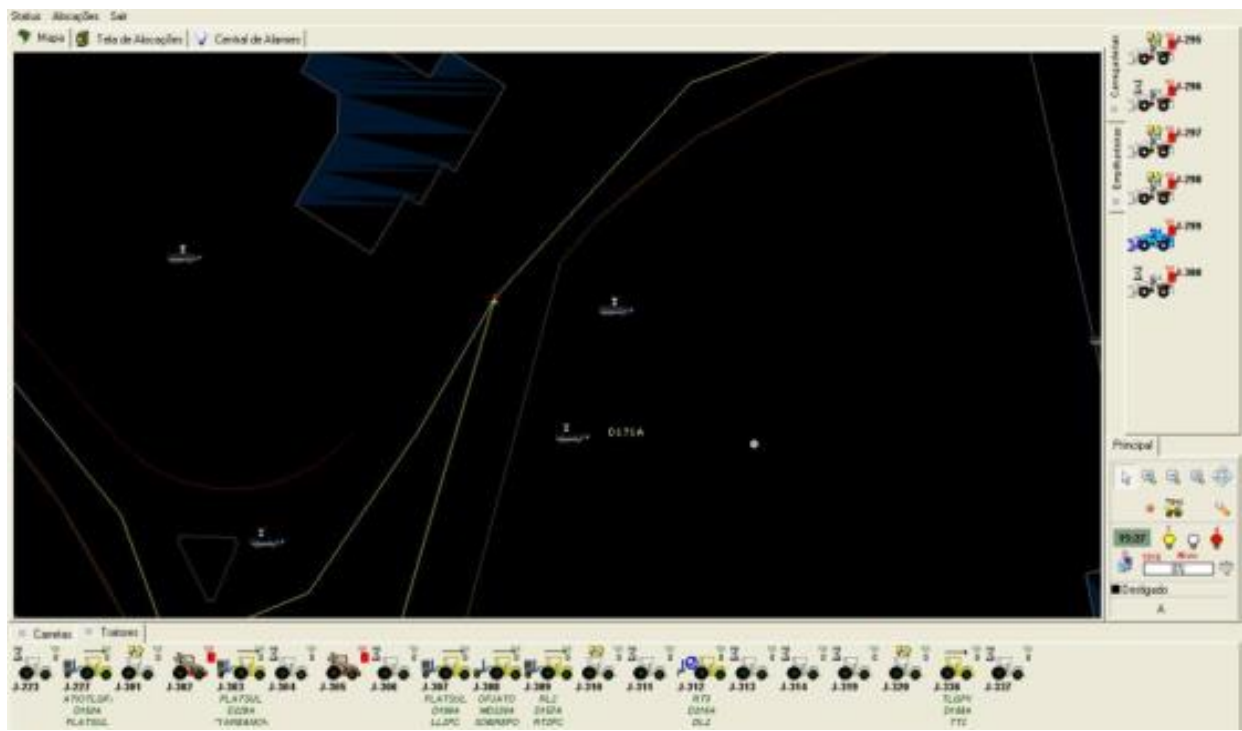

Figura 2 - Tela anterior da central de operações

Em função de todos os fatores acima, somado o período de nova contratação do operador logístico, foram realizadas várias analises de mercado, visita em clientes atuais das empresas concorrentes e iniciado a parceria entre a VSB e DDMX para implantação de um único sistema para gestão de toda a frota de equipamentos logísticos, incluindo todos os tipos de equipamentos como: carretas, tratores, empilhadeiras, pás carregadeiras, caminhões, paleteiras e outros, possibilitando uma completa gestão dos recursos.

\section{RESULTADOS E DISCUSSÃO}

O processo de definição das necessidades, metodologia de analise, escolha de hardwares, estruturação das centrais de operação da VSB e elaboração dos relatórios duraram cerca de 5 meses, e atualmente ainda são realizadas diversas melhorias e adaptações a novos cenários.

\subsection{Funcionalidades do Sistema}

Para atendimento as demandas da VSB foram criadas 3 principais a estrutura (figura 3), onde existem aplicações e centrais especificas para cada atividade e equipamentos, conforme cada particularidade. 


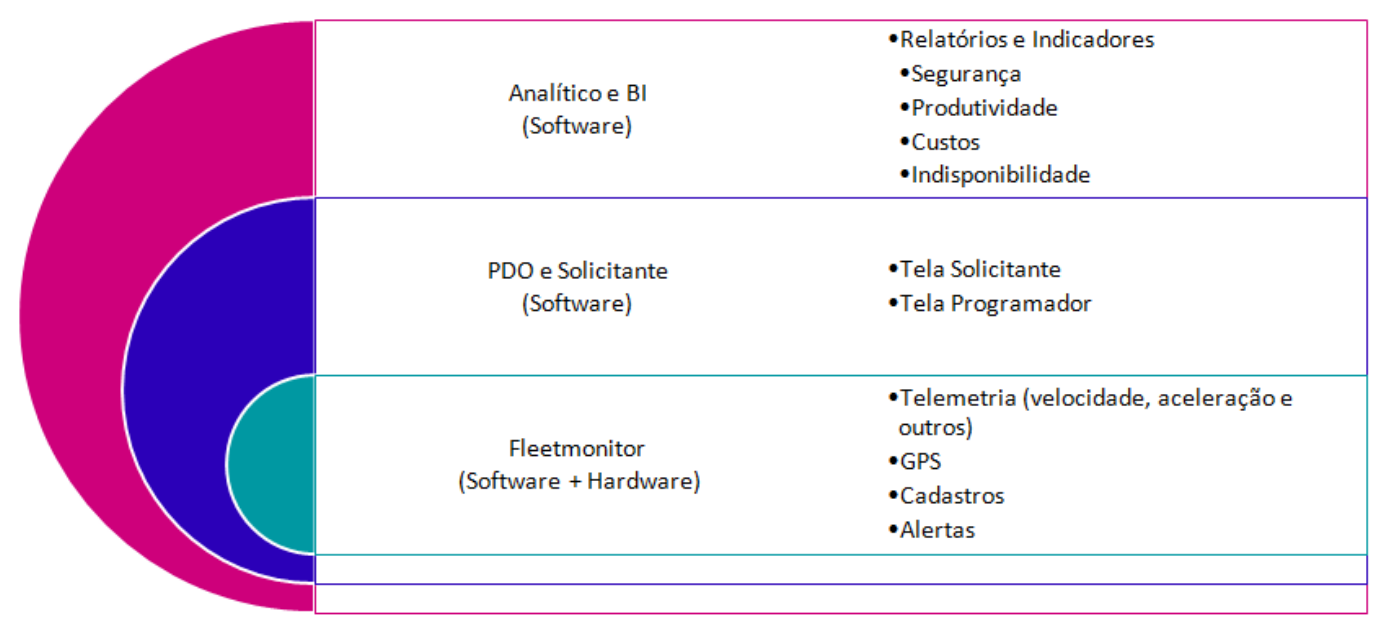

Figura 3 - Estrutura do sistema de gestão

Abaixo descrição das principais funcionalidades de cada funcionalidade atual do sistema de forma resumida para os tópicos: Frotas; PDO e Solicitante; Analítico e BI.

\subsubsection{Fleetmonitor}

Este ambiente é à base de todas as informações, onde são coletados e registrados os dados apurados através de telemetria e comunicação de dados através da tecnologia $3 G$ e $4 G$, onde para a implementação do case apresentado foi realizado pela DDMX uma avaliação da cobertura de todas as operadores e avaliado a que atendia com melhor cobertura e aderência a necessidade da VSB.

Atualmente são utilizados hardwares de mercado e de fácil substituição (figura 4), que são de responsabilidade do prestador de serviço sua aquisição, manutenção e controle devido a operação ser realizada por $100 \%$ da equipe do prestador. Um grande diferencial deste modelo é que não existe uma restrição com relação ao uso de qualquer outra tecnologia de rastreamento de mercado.
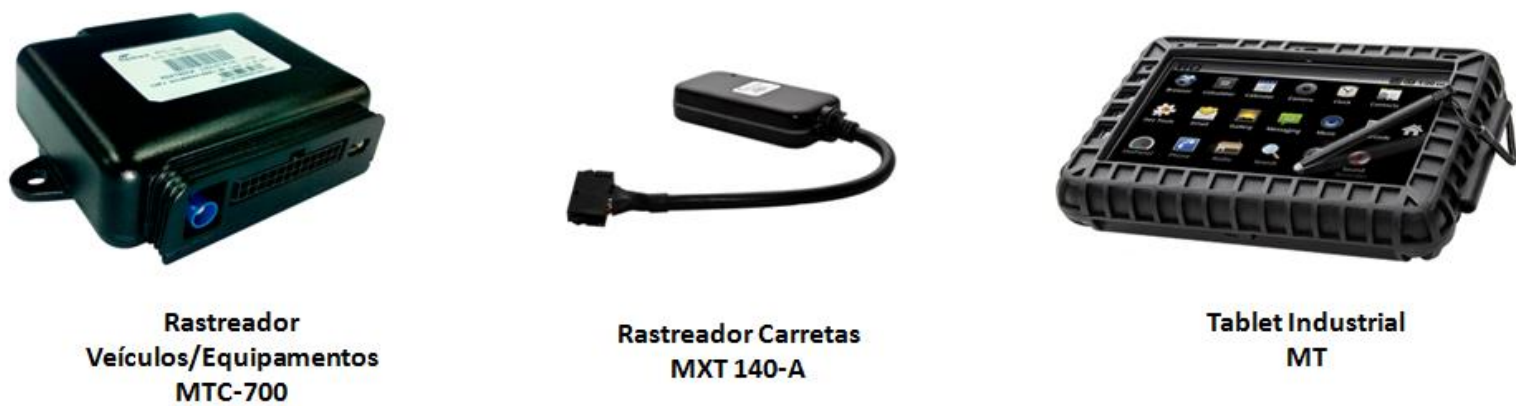

Figura 4 - Hardwares utilizados

Com base em todos os dados apurados pelos rastreadores instalados nos equipamentos, e todas as configurações que são realizadas de forma simples e variável no ambiente (figura 5) é possível controlar todos os acessos as áreas 
mapeadas, controles de velocidade, telemetria dos equipamentos, alertas de permanência e analise de históricos de movimentação.

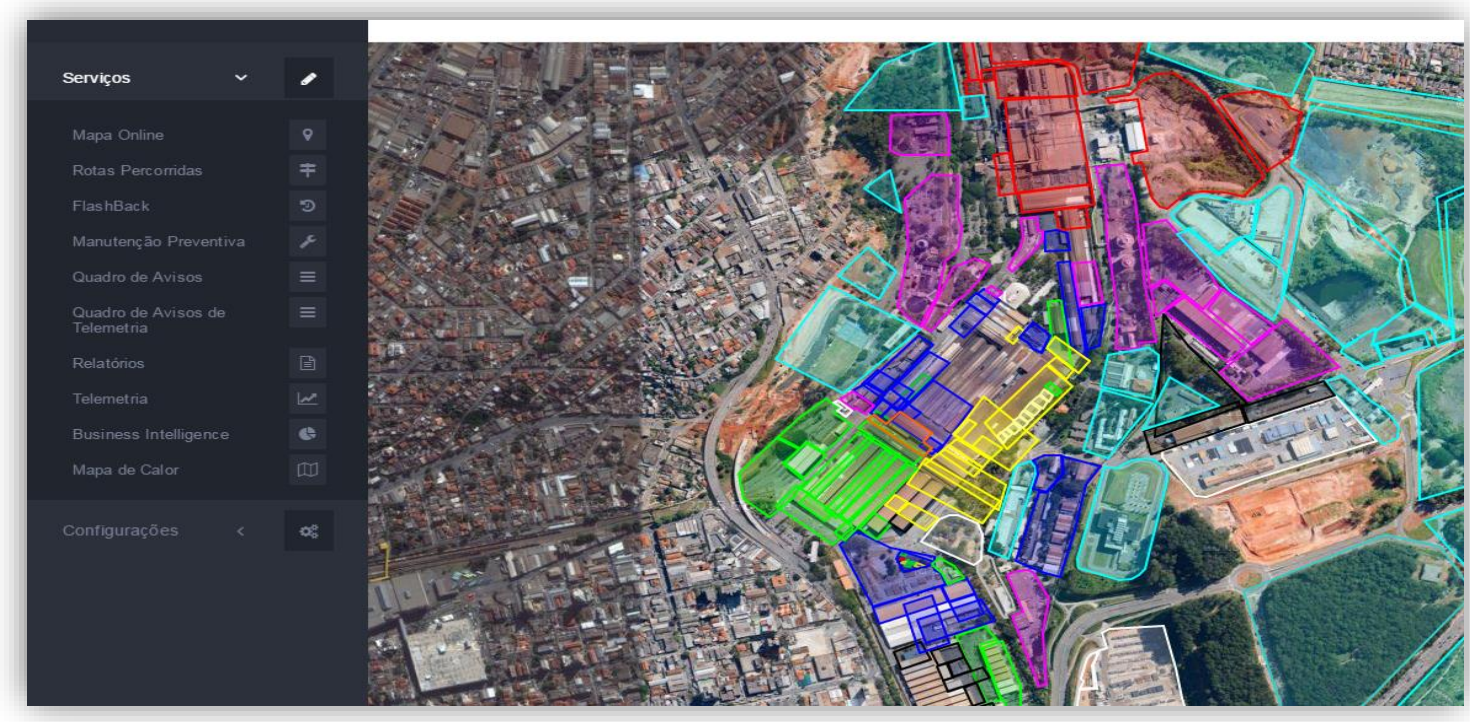

Figura 5 - Tela principal do fleetmonitor

Vale destacar as seguintes funcionalidades:

- Mapa on-line dos equipamentos através de acesso via internet em computadores e smartphones;

- Histórico de fácil acesso de rotas percorridas, equipamentos que acessaram determinada área, mapa de calor e outras funcionalidades para a gestão;

- Cadastro de operadores conforme equipamentos específicos e possibilidade de controlar a operação do mesmo;

- Cadastro de áreas com características especifica como: velocidade, tempo de permanência, alertas de entrada e saída da área de forma fácil e com alertas automáticos por e-mail;

- Relatórios pré-definidos com possibilidade de agendamento e envio automático conforme programação do usuário;

- Controle de telemetria conforme sensores definidos para cada equipamento. Exemplo: Controle de acionamento da "patola" para acionamento do munck e/ou bruck, sensor de acoplamento/desacoplamento das carretas e tratores e outros;

- Controles de alerta de percurso, parametrizado conforme demanda;

\subsubsection{PDO (programador) e Solicitante}

De acordo com as características das operações de cada equipamento foram criados alguns ambientes e métodos para as solicitações de equipamentos pelos usuários, definição e estratégia dos recursos que irão atender conforme 0 programador de logística e outras funcionalidades (figura 6). 


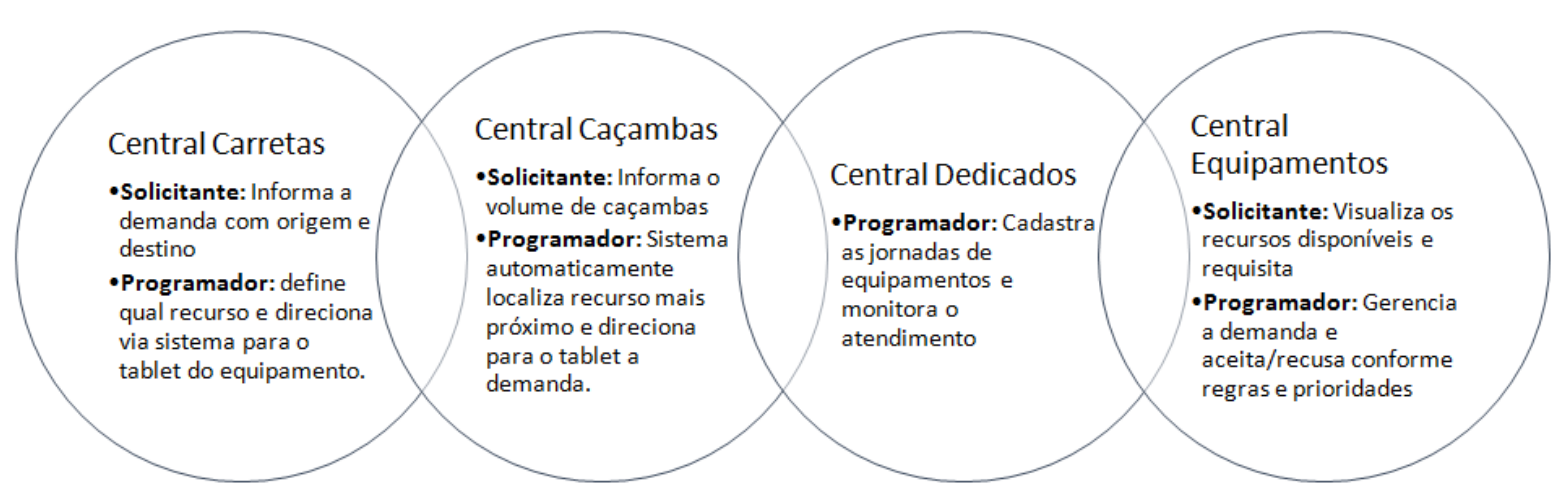

Figura 6 - Estrutura básica dos Solicitantes e PDO

Com isso foram criadas telas especificas para cada central e de fácil acesso para os solicitantes, onde é definido através do cadastro de usuários o acesso a cada tipo de central, sendo possível acompanhar em tempo real o atendimento a requisição especifica.

Vale destacar as seguintes funcionalidades:

- Sistema de fácil interação entre usuários e programadores;

- Acompanhamento da situação da demanda;

- Visualização on-line dos recursos;

- Sem limites de usuários;

- Acesso via internet em computadores e smartphones;

- Adequação a política de segurança da informação da empresa.

\subsubsection{Analítico e BI (Business Intelligence)}

Todas as informações coletadas pela estrutura Fleetmonitor e as demandas do Solicitante e PDO, são consolidados em um único ambiente, onde é possível interligar as informações, interligando a realidade (GPS e Telemetria) com as demandas (requisições e alocações), gerando as analises necessárias para os indicadores de atendimento e gestão de contratos.

Este ambiente pode ser mapeado conforme demanda, onde é definido o acesso de cada relatório conforme o usuário, e é utilizado para os controles dos indicadores de disponibilidade contratual previsto para cada tipo de equipamento.

Vale destacar as seguintes funcionalidades:

- Foram definidos relatórios de produtividade especifico de cada equipamento conforme a telemetria e sensores instalados;

- Foram determinadas as tolerâncias de ausência especifica de cada recurso para apuração correta da taxa de indisponibilidade;

- Elaboração de diversos relatórios para apoiar as analises como: indisponibilidade, produtividade e segurança;

- Integração com outros sistemas da VSB como SAP; 
- Elaboração de ranking dos operadores conforme critérios internos de atendimento, segurança e produtividade;

- Sistema de BI interativo e com diversos indicadores mapeados e de fácil entendimento da operação (figura 7).

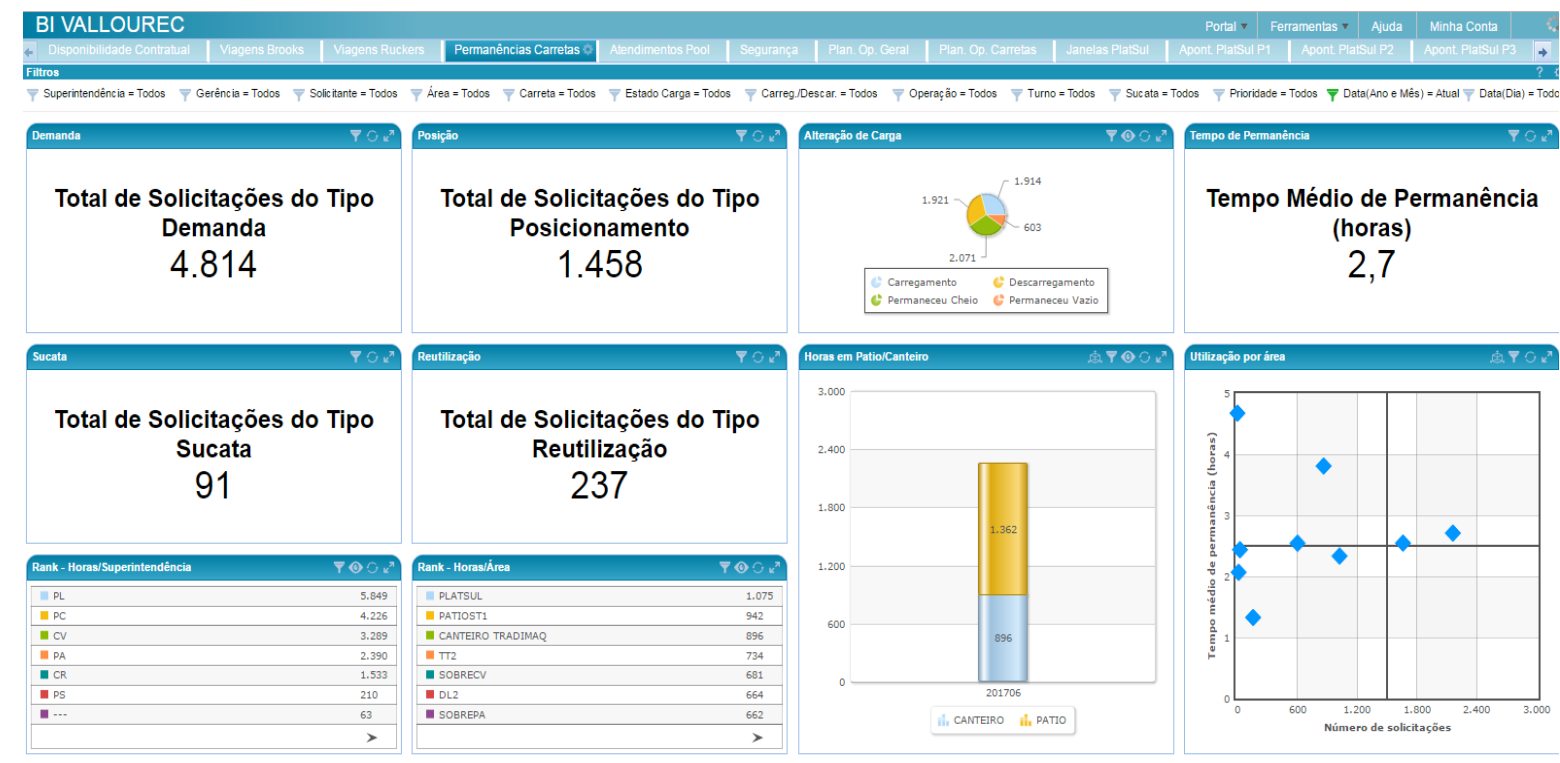

Figura 7- Modelo BI

Em todo o processo definição do sistema, desenvolvimento da ferramenta e todas as alterações de demandas necessárias à parceria entre VSB e DDMX ocorreu de forma coerente com o contrato, reforçando desta forma a parceria entre as empresa, e resguardando a aderência do sistema a nossa necessidade de acordo com todas as alterações de processo ao longo da implantação do modelo e as inovações tecnológicas.

\section{CONCLUSÃO}

Para uma gestão de contrato de forma adequada as necessidades técnicas, de segurança e produtividade, são essenciais à utilização de uma ferramenta que apoia as analise e tomadas de decisão. O modelo atual de rastreamento e gestão da frota atende além de todo o processo técnico, tem como característica uma grande aderência aos controles de segurança solicitados pela empresa sendo utilizados por diversas vezes de forma preventiva e nas analises de incidentes.

Por ser um sistema de fácil operação, com tecnologia de mercado e com ferramentas amigáveis, não tivemos dificuldades em sua implantação entre os usuários e programadores, e o operador logístico entende o beneficio do sistema e garante a sua utilização.

Neste momento estamos analisando outras inovações como aplicação de leitura de carga das carretas, sinais sonoros e cronômetros, dispositivos de sensores de presença para o processo de carregamento, automatização do processo de medição do contrato de acordo com operação de recursos e integração com SAP para 
faturamento, entre outros. No ano de 2016 finalizamos a parceria com a DDMX para modelo similar na gestão de frota de transporte de pessoal dos funcionários.

E principalmente conseguimos atender a premissa de monitoramento de $100 \%$ da frota interna, sem aumento de custo com relação ao modelo anterior e com flexibilidade de aumento de recursos a serem gerenciados.

\section{REFERÊNCIAS}

1 LAUDON \& LAUDON, Kenneth C; Jane P (2007). "Sistemas de Informação Gerenciais". $7^{a}$ edição. Tradução Thelma Guimarães; Revisão técnica Belmiro N. João. Editora Pearson Prentice Hall, São Paulo - SP.

2 REZENDE, Denis Alcides (2008). "Planejamento estratégico para organizações privadas e publicas: guia pratico para elaboração do projeto do plano de negócios". Editora Brasport, Rio de Janeiro - RJ.

3 ESSS. Rio de Janeiro. OS PILARES DA INDÚSTRIA 4.0. 2017. Disponível em http://www.esss.com.br/blog/2017/01/os-pilares-da-industria-4-0/>. Acesso em 05/03/2017. 